\title{
Brain activation of the defensive and appetitive survival systems in obsessive compulsive disorder
}

\author{
Óscar F. Gonçalves • José Miguel Soares • Sandra Carvalho • Jorge Leite • Ana Ganho • \\ Ana Fernandes-Gonçalves • Brandon Frank • Fernando Pocinho • João Relvas • \\ Angel Carracedo • Adriana Sampaio
}

(C) Springer Science+Business Media New York 2014

\begin{abstract}
Several studies have shown that basic emotions are responsible for a significant enhancement of early visual processes and increased activation in visual processing brain regions. It may be possible that the cognitive uncertainty and repeated behavioral checking evident in Obsessive Compulsive Disorder (OCD) is due to the existence of abnormalities in basic survival circuits, particularly those associated with the visual processing of the physical characteristics of emotional-laden stimuli. The objective of the present study was to test if patients with OCD show evidence of altered basic survival circuits, particularly those associated with the visual processing of the physical characteristics of emotional stimuli. Fifteen patients with OCD and 12 healthy controls underwent functional magnetic resonance imaging acquisition
\end{abstract}

Ó. F. Gonçalves $(\bowtie) \cdot$ S. Carvalho $\cdot$ J. Leite $\cdot$

A. Fernandes-Gonçalves $\cdot$ B. Frank

Department of Counseling and Applied Educational Psychology,

Bouvé College of Health Sciences, Northeastern University,

404 Int'l Village, Boston, MA 02115, USA

e-mail: o.goncalves@neu.edu

Ó. F. Gonçalves · S. Carvalho · J. Leite · A. Ganho • A. Sampaio Neuropsychophysiology Laboratory, CIPsi, School of Psychology, University of Minho, Braga, Portugal

J. M. Soares

Life and Health Sciences Research Institute, University of Minho, Braga, Portugal

J. M. Soares

ICVS-3Bs PT Government Associate Laboratory, Braga, Guimarães, Portugal

F. Pocinho $\cdot$ J. Relvas

Department of Psychiatry, University of Coimbra Hospitals, Coimbra, Portugal

A. Carracedo

Forensic Genetics Unit, Institute of Legal Medicine, Faculty of Medicine, University of Santiago de Compostela, Galicia, Spain while being exposed to emotional pictures, with different levels of arousal, intended to trigger the defensive and appetitive basic survival circuits. Overall, the present results seem to indicate dissociation in the activity of the defense and appetitive survival systems in OCD. Results suggest that the clinical group reacts to basic threat with a strong activation of the defensive system mobilizing widespread brain networks (i.e., frontal, temporal, occipital-parietal, and subcortical nucleus) and blocking the activation of the appetitive system when facing positive emotional triggers from the initial stages of visual processing (i.e., superior occipital gyrus).

Keywords Obsessive-compulsive disorder · Survival circuits $\cdot$ Neuroimaging

\section{Introduction}

In order to potentiate the appropriate survive-thrive strategies; organisms are required to have systems that allow a fast and accurate perception of the physical characteristics of the environment. In most species, particularly mammals, basic emotions can be defined as survival brain circuits by which individuals increase perceptual alertness of their environment in order to reduce uncertainty while responding to relevant stimuli triggers (LeDoux 2012). Two survival systems have been described: appetitive and defensive (please see for review Lang and Bradley 2013). Most interestingly, at the brain level, the two motivational systems with survival driven functions seem to overlap (Sabatinelli et al. 2011). Particularly interesting is the common finding of a significant enhancement of early visual processes such as contrast sensitivity (Phelps et al. 2006) along with an increased activation in visual areas, particularly in those associated with processing the physical characteristics of the stimuli (i.e., ventral visual stream) (Wright et al. 2004). Several authors have argued that this 
increase in visual processing in emotional situations is supported by current neuroanatomical evidence of amygdala projections towards ventral stream visual areas, both at the structural (Amaral et al. 2003) and functional levels (Williams et al. 2006). There is now evidence that amygdala - extra striate functional connectivity is particularly evident while processing novel pleasant or unpleasant emotional stimuli (Wendt et al. 2011) and that the early detection of emotional relevance may be particularly associated with a process of reentrant feedback between ventral stream visual regions (i.e., inferotemporal cortex) and the amygdala (Sabatinelli et al. 2009).

At the evolutionary level, the facilitation of perceptional processing by emotional priming may allow a quicker and more efficient activation of either the organism's defensive (i.e., fight or flight response) or appetitive systems (i.e., ingestion, copulation, and care giving) (Bradley et al. 2001). On the contrary, a decrease in perceptual sensitivity to significant environmental triggers can be associated with increased feelings of uncertainty and doubt. In order to reduce uncertainty, the individual entails a process of repeated checking. However this repeated checking ends up, paradoxically, reducing memory confidence, triggering increased feelings of uncertainty and doubt and motivating further checking (Radomsky and Alcolado 2010). This may be what happens in the case of obsessive-compulsive disorder (OCD), a mostly disabling anxiety disorder, characterized by difficulty in inhibiting repetitive thoughts (obsessions) and stereotyped behaviors (compulsions). Even though OCD is a complex disorder with a variety of symptomatic configurations (e.g., symmetry and ordering; forbidden thoughts; contamination and cleaning), prevalent thoughts of uncertainty and the urge for repeated behavioral checking seem to be the most common associated characteristics (Bloch et al. 2008).

There is now abundant evidence of structural and functional brain abnormalities in OCD patients. Morphometric gray matter abnormalities in regions associated with the frontalsubcortical loops have been consistently found (Rotge et al. 2010). Additionally, functional neuroimaging studies demonstrate that the provocation of OCD symptoms is associated with an activation of the orbitofrontal and anterior cingulated loops (Rotge et al. 2008). Effective therapy tends to normalize the hyper activation of these frontal-subcortical pathways as well as increasing the activity of secondary visual regions (e.g., fusiform gyrus) (Yamanishi et al. 2009).

Several studies have found evidence for decreased activation of the visual cortex (i.e., inferior occipital) in patients with OCD when confronted with disgust inducing pictures (Shapira et al. 2003). In accordance, evidence of specific visual processing deficits in OCD, namely in terms of visual organization (Rampacher et al. 2010), and perception of biological movement (Kim et al. 2008) has been documented, and suggesting alterations at the visual processing level.
In sum, there is now evidence that presentation of emotional content is responsible for an increased activation of extra striate and ventral stream visual areas. It may be possible that the cognitive uncertainty and repeated behavioral checking evident in OCD may be due to the existence of abnormalities in basic survival circuits, particularly those associated with the visual processing of the physical characteristics of emotional stimuli.

In the current study, we investigated if patients with OCD $(n=15)$, when compared with healthy controls $(n=12)$, show evidence of abnormalities in the blood oxygen level dependent (BOLD) functional magnetic resonance imaging (fMRI) brain response to basic emotional pictures, with different levels of arousal (high arousal and low arousal), intended to trigger the defensive (unpleasant stimuli) and appetitive (pleasant stimuli) basic survival circuits.

\section{Methods}

\section{Participants}

Fifteen patients with OCD and twelve healthy controls (matched by age, gender and education) were enrolled in the present study. Both OCD patients and healthy controls were right-handed individuals, as assessed by the Edinburgh Handedness Inventory (EHI $\geq 80$ ) (Oldfield 1971). The OCD individuals were diagnosed with the Structured Clinical Interview for Diagnostic and Statistical Manual of Mental Disorders (SCID-I) (First et al. 1996) and OCD symptom severity was assessed using the "The Yale-Brown Obsessive Compulsive Scale" (YBOCS) (Goodman et al. 1989). Depression symptoms were assessed using the Beck Depression Inventory (BDI) (Beck et al. 1988). Comorbidity was restricted to OCD spectrum disorders. Patients with OCD were referred after diagnosis by an experienced clinician (psychiatrist or clinical psychologist) and subsequently assessed by a trained clinician using the SCID-I, YBOCS, and BDI. No comorbid conditions were identified in any participants from the OCD group. All of the patients were currently on stable pharmacological treatment and behavioral therapy. The control group consisted of individuals without history of drug abuse, psychiatric or neurological disorder and use of psychotropic medication. The healthy control group underwent the same assessment as the OCD participants.

Demographic information and symptom severity data are presented in Table 1.

Stimuli

Fifty pictures were selected from the database of International Affective Picture System (IAPS) (Bradley et al. 2006) intended to activate (i.e., high and low activation) the different 
Table 1 Demographic information by group

\begin{tabular}{lll}
\hline & OCD & Healthy controls \\
\hline Number & 15 & 12 \\
\% Female & 26.7 & 33.3 \\
Age & $31.67(11.44)$ & $30.92(8.98)$ \\
\% Caucasian & 100 & 100 \\
Highest education level & & \\
$\quad$ \% Less than high school & 33.3 & 16.7 \\
$\quad$ \% High school & 40.0 & 41.7 \\
$\quad$ \% College degree & 26.7 & 41.7 \\
EHI & $93.53(7.86)$ & $90.0(14.77)$ \\
YBOCS* & $23.80(6.74)$ & $.67(1.23)$ \\
BDI** & $9.47(6.63)$ & $3.33(3.17)$ \\
\hline
\end{tabular}

$O C D$ obsessive-compulsive disorder, $E H I$ edinburgh handedness inventory, YBOCS yale-brown obsessive compulsive scale, $B D I$ beck depression inventory

${ }^{*}$ Group differences significant at $p<.001 ; * * p<.01$ [YBOCS, t $(15.16)=$ 13.02, $\mathrm{d}=4.77$; BDI, $\mathrm{t}(20.97)=3.16, \mathrm{~d}=1.18]$

survival systems (i.e., defensive and appetitive). In IAPS the valence scale ranges from 1 (highly unpleasant) to 9 (highly pleasant) and the arousal scale ranges from 1 (no arousal at all) to 9 (high arousal). More specifically, 10 high arousal pleasant IAPS images [erotic: valence $\mathrm{M}=6.81(\mathrm{SD}=0.42)$, arousal $\mathrm{M}=6.51(\mathrm{SD}=0.36)]$ were used to trigger high activation of the appetitive system; 10 high arousal unpleasant [mutilation: valence $1.69(0.25)$, arousal $6.70(0.38)]$ for high activation of the defensive system; 10 low arousal pleasant for low activation of the appetitive system [valence $7.63(0.33)$, arousal 4.23 (0.36)]; 10 low arousal unpleasant for low activation of the defensive system [valence $3.58(0.45)$, arousal $4.10(0.40)$ ]; and 10 emotional neutral objects [valence $5.06(0.29)$, arousal $2.81(0.56)]$.

The following IAPS pictures were used in the present study: High Activation of the Defensive System: 3010, 3060, 3064, 3068, 3069, 3100, 3102, 3150, 3168, 3266; Low Activation of the Defensive System: 2100, 2110, 2271, 2278, 2312, 2399, 2455, 2490, 2491, 9331; Neutral: 7025, 7042, 7052, 7053, 7059, 7090, 7100, 7150, 7175, 7235; High Activation of the Appetitive System: 4643, 4647, 4652, 4656, 4659, 4660, 4670, 4680, 4687, 4800; Low Activation of the Appetitive System: 2050, 2091, 2154, 2165, 2304, 2310, 2311, 2332, 2360, 2540.

Pictures were matched in terms of brightness, contrast, mean spatial frequency and $90 \%$ quality JPEG. The stimuli were presented in event-related fMRI design (spaced mixed trial), consisting of one $10 \mathrm{~s}$ epoch containing a fixator cross (black cross on a white background) immediately followed by one $20 \mathrm{~s}$ epoch with an emotional-laden picture (High Activation of the Defensive System; Low Activation of the Defensive System; Neutral; High Activation of the Appetitive
System and Low Activation of the Appetitive System). Stimuli were presented in two separate runs (25 stimuli for each run -5 stimuli per condition). All the presentations were randomized across participants, and within each run. Participants were instructed that different pictures were going to be presented on the screen and that they should be comfortably focusing on the cross at the center of the pictures. No response was required from the participants.

Image data acquisition

Within 1 week after clinical assessment, participants underwent the scanning procedure in a clinically approved Siemens Magnetom TrioTim 3 T (Siemens Medical Solutions, Erlangen, Germany) using the Siemens 12-channel receiveonly head coil. E-Prime $2.0{ }^{\circledR}$ (Psychology Software Tools Inc.) was used to program the fMRI paradigm and the visual stimuli were presented using an LCD projector Silent Vision Model SV-6011 (Avotec, Inc.).

For structural analysis and registration to standard space, a T1 high-resolution anatomical sequence, a 3D MPRAGE (magnetization prepared rapid gradient echo) was performed with the following scan parameters: repetition time (TR) $=$ $2.3 \mathrm{~s}$, echo time $(\mathrm{TE})=2.98 \mathrm{~ms}, 160$ sagittal slices with no gap, Field-of-View $(\mathrm{FoV})=256 \mathrm{~mm}$, flip angle $(\mathrm{FA})=9^{\circ}$, inplane resolution $=1 \times 1 \mathrm{~mm}^{2}$ and slice thickness $=1 \mathrm{~mm}$. The scanning parameters for fMRI acquisition were the following: $\mathrm{TR}=3 \mathrm{~s}, \mathrm{TE}=39 \mathrm{~ms}, \mathrm{FA}=90^{\circ}$, in-plane resolution and slice thickness $3 \mathrm{~mm}, 41$ ascending interleaved axial slices with no gap and $\mathrm{FoV}=256 \mathrm{~mm}$.

\section{Data preprocessing and analysis}

Before any data processing and analysis, structural and functional acquisitions were visually inspected for critical head motion and brain lesions. The fMRI data preprocessing was performed using Statistical Parametric Mapping version 8 (SPM8 ${ }^{\circledR}$ ) (http://www.fil.ion.ucl.ac. $\mathrm{uk} / \mathrm{spm}$ ). Images were corrected for slice-timing, motion with realignment processing, spatially transformed to the standard MNI (Montreal Neurologic Institute) coordinates, resampled to $2 \times 2 \times 2 \mathrm{~mm}^{3}$, smoothed with an 8 $\mathrm{mm}$ full-width half-maximum Gaussian kernel and high pass temporal filtered (filter width of $128 \mathrm{~s}$ ).

The fMRI paradigm statistical analyses were performed using a general linear model (GLM) approach with seven regressors for the different picture visualization conditions, which were convolved with the hemodynamic response function. The regressors created were: high and low activation of the appetitive, defensive, and neutral pictures, and the differences among these different conditions. For group comparisons, two-sided one-sample and two-sample t-tests were used. For group comparisons, two-sided one-sample and two- 
Table 2 Regions activated by the defensive and appetitive systems in OCD and healthy controls

\begin{tabular}{|c|c|c|c|c|c|}
\hline Condition & Regions & $\mathrm{R} / \mathrm{L}$ & Peak MNI coordinate & Cluster size & $\begin{array}{l}\text { Maximum } \\
\text { Z-score }\end{array}$ \\
\hline \multirow[t]{9}{*}{ Defensive system condition (OCD > healthy controls) } & Putamen & $\mathrm{L}$ & $28,2,8$ & 575 & 3.88 \\
\hline & Precentral gyrus & $\mathrm{L}$ & $-36,6,42$ & 1,004 & 3.86 \\
\hline & Frontal inferior triangularis & $\mathrm{L}$ & $-54,20,18$ & & 3.09 \\
\hline & Frontal inferior opercularis & $\mathrm{R}$ & $54,16,16$ & 1,263 & 3.56 \\
\hline & Putamen & $\mathrm{R}$ & $34,4,0$ & & 3.34 \\
\hline & Middle Temporal gyrus & $\mathrm{R}$ & $46,-20,-8$ & 305 & 3.55 \\
\hline & Superior temporal gyrus & $\mathrm{R}$ & $60,-18,-6$ & & 3.19 \\
\hline & Medial frontal orbitofrontal gyrus & $\mathrm{L}$ & $-6,54,-8$ & 82 & 3.32 \\
\hline & Postcentral gyrus & $\mathrm{R}$ & $64,-18,42$ & 282 & 3.22 \\
\hline Appetitive system condition (healthy controls $>$ OCD) & Superior occipital gyrus & $\mathrm{L}$ & $-12,-92,34$ & 186 & 3.38 \\
\hline
\end{tabular}

sample t-tests were used, with random effect analysis. Based on the chosen threshold for functional data, results were considered significant at a corrected for multiple comparisons $p<0.05$ threshold (based on the combination of $p<0.01$ with a minimum cluster size of 71 voxels), determined by a Monte Carlo simulation program (AlphaSim). Then, we used a corrected threshold Zscore of 3.09. The AAL gray matter mask (TzourioMazoyer et al. 2002) was used to calculate the Monte Carlo correction and as an inclusive mask in all studied contrasts. The description of all the statistics and brain coordinates for the different activation areas in the distinct conditions tested are presented in Tables 2 and 3.

\section{Results}

The neuroimaging data will be presented in four sections. The first two sections will present the results of the whole-brain analysis comparing OCD patients and healthy controls in the activation of the defensive and the appetitive system when contrasted with the emotional neutral activation (independently of the level of activation). In the third and fourth section identical contrasts will be presented, but this time only for the high activation conditions (for both defensive and appetitive systems).

Differences between OCD and healthy controls for the activation of the defensive system

When presented with stimuli (i.e., mutilation IAPS images) triggering the activation of the defensive system, OCD patients, when compared with healthy controls, were found to have significant increases in the following brain regions (see Fig. 1 and Table 2): left inferior frontal gyrus (triagularis); right inferior frontal gyrus (opercularis), left medial orbitofrontal gyrus, bilateral putamen, right superior temporal gyrus, right middle temporal gyrus and right postcentral gyrus.

Differences between OCD and healthy controls for the activation of the appetitive system

When compared to healthy controls, patients with OCD showed significantly less activation in the appetitive system condition, only on the superior occipital gyrus. No significantly increased activations were found anywhere in the brain for the OCD group (see Fig. 1 and Table 2).

Table 3 Regions with significant increases during high activation of the defensive and appetitive systems in OCD and healthy controls

\begin{tabular}{|c|c|c|c|c|c|}
\hline Condition & Regions & $\mathrm{R} / \mathrm{L}$ & Peak MNI coordinate & Cluster size & $\begin{array}{l}\text { Maximum } \\
\text { Z-score }\end{array}$ \\
\hline \multirow{2}{*}{$\begin{array}{l}\text { High activation of defensive system condition } \\
\qquad(\mathrm{OCD}>\text { healthy controls })\end{array}$} & Medial frontal orbitofrontal gyrus & $\mathrm{L}$ & $2,56,-10$ & 85 & 3.40 \\
\hline & Postcentral gyrus & $\mathrm{L}$ & $-56,-20,14$ & 265 & 3.20 \\
\hline \multirow{4}{*}{$\begin{array}{l}\text { High activation of appetitive system condition } \\
\text { (healthy controls }>\text { OCD) }\end{array}$} & Precentral gyrus & $\mathrm{R}$ & $48,-8,60$ & 337 & 3.55 \\
\hline & Paracentral lobule & $\mathrm{L}$ & $54,-2,52$ & & 3.34 \\
\hline & Superior occipital gyrus & $\mathrm{L}$ & $-12,-90,40$ & 401 & 3.48 \\
\hline & Fusiform gyrus & $\mathrm{L}$ & $-26,-50,-16$ & 189 & 3.27 \\
\hline
\end{tabular}


Fig. 1 a Regions where OCD patients, when compared with healthy controls, showed increased activation for the defensive system condition; b Regions where participants from the healthy controls group, when compared with OCD, showed increased activation in the appetitive system condition

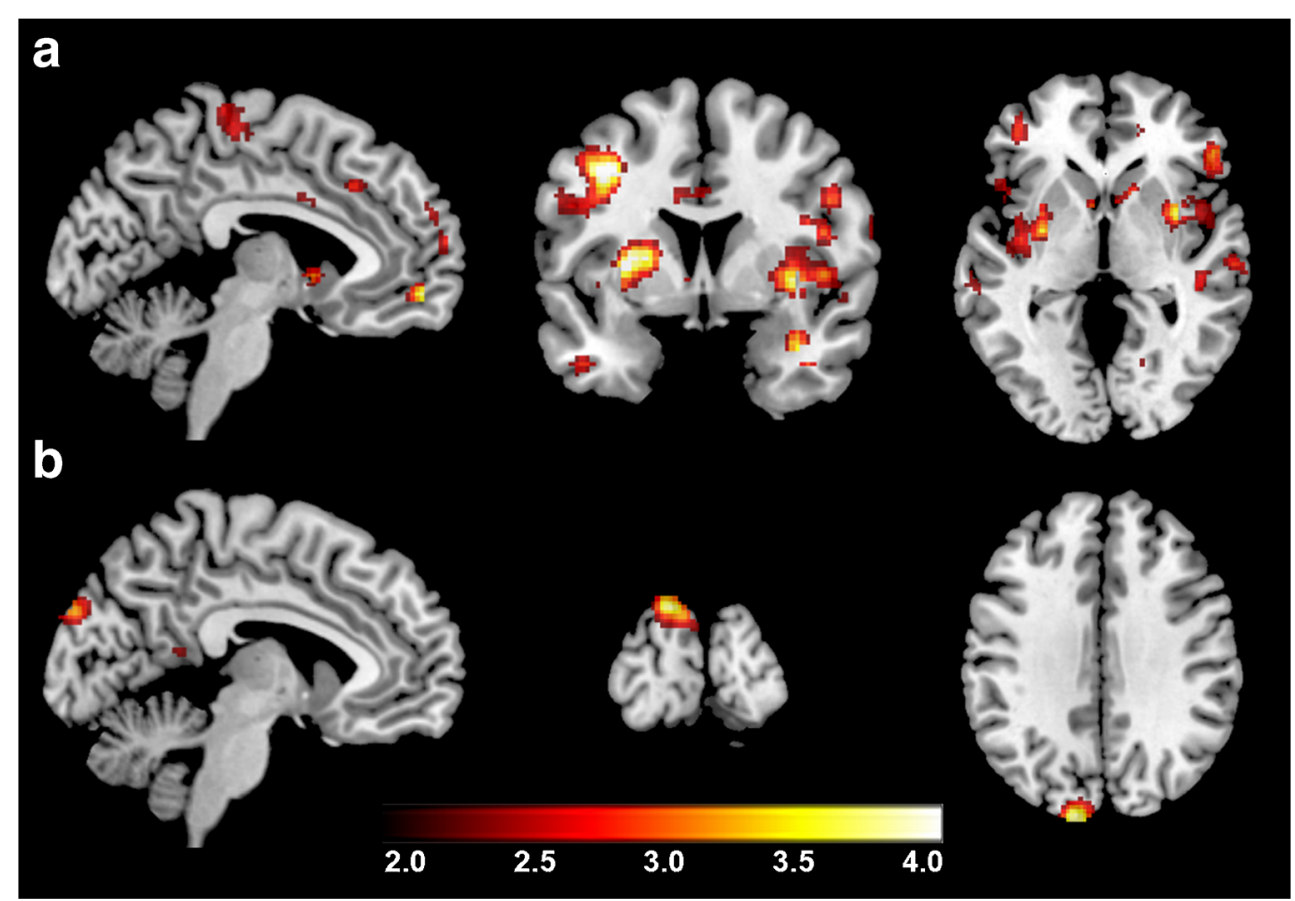

Differences between OCD and healthy controls for high activation of the defensive system

When high activation of the defensive systems was isolated and contrasted with the neutral condition, we found that patients with OCD had significant increases in the activation of the left medial orbitofrontal gyrus and left postcentral gyrus (see Fig. 2 and Table 3).

Differences between OCD and controls for high activation of the appetitive system

When high activation of the appetitive system was isolated and contrasted with the neutral condition (see Fig. 2 and Table 3), patients with OCD showed significant decreases in activity for multiple brain regions, such as the right pre-central gyrus, left para central lobule, left superior occipital gyrus, and left fusiform gyrus.

\section{Discussion}

The objective of the present study was to test whether patients with OCD show evidence of altered basic survival circuits, particularly those associated with the visual processing of the physical characteristics of emotional stimuli. More specifically, we were interested in knowing if the fronto-subcortical activation consistently found in OCD was correlative of a deactivation of occipital/parietal regions associated with visual-perceptual processing of stimuli triggering, with different levels of arousal (high arousal and low arousal) the defensive (unpleasant stimuli) and appetitive (pleasant stimuli) basic survival circuits.

Overall, the results showed that the brain activation pattern was different according to the basic survival system being activated (defensive or appetitive), but also between groups (OCD patients and healthy controls). More specifically, OCD patients tend to respond to the activation of the defensive system recruiting a distributed brain network, involving frontal (e.g., left inferior frontal gyrus (pars triagularis); right inferior frontal gyrus (pars opercularis), left medial orbitofrontal gyrus, left pre central gyrus), temporal (e.g., right superior temporal gyrus, right middle temporal gyrus), parietal (e.g., right postcentral gyrus) and subcortical nucleus (e.g., bilateral putamen). These findings seem to overlap with those found when only the high activation of the defensive system condition is compared to the neutral condition, with increased activation in patients with OCD (frontal regions-left medial orbitofrontal gyrus) and parietal regions (left postcentral gyrus).

These findings of widespread brain activation in OCD are consistent with conclusions derived from various metaanalyses of experiments involving the provocation of symptoms. For example, Rotge et al. (2008), on a quantitative voxel-based meta-analysis of the functional studies (fMRI and PET), and using a symptom provocation paradigm, reported evidence for the hyperactivation of cortico-striatopallido-thalamo-cortical circuits, but also other regions such as the superior temporal gyrus, precuneus, and dorsolateral prefrontal cortex. On a more extended and inclusive narrative review (all types of functional studies), Del Casale et al. (2011), reported a complex picture involving dysfunction 
Fig. 2 a Regions where OCD patients, when compared with healthy controls, showed increased activation in the High Activation of the defensive system condition; b Regions where healthy controls, when compared with OCD patients, showed increased activation during High Activation of the appetitive system
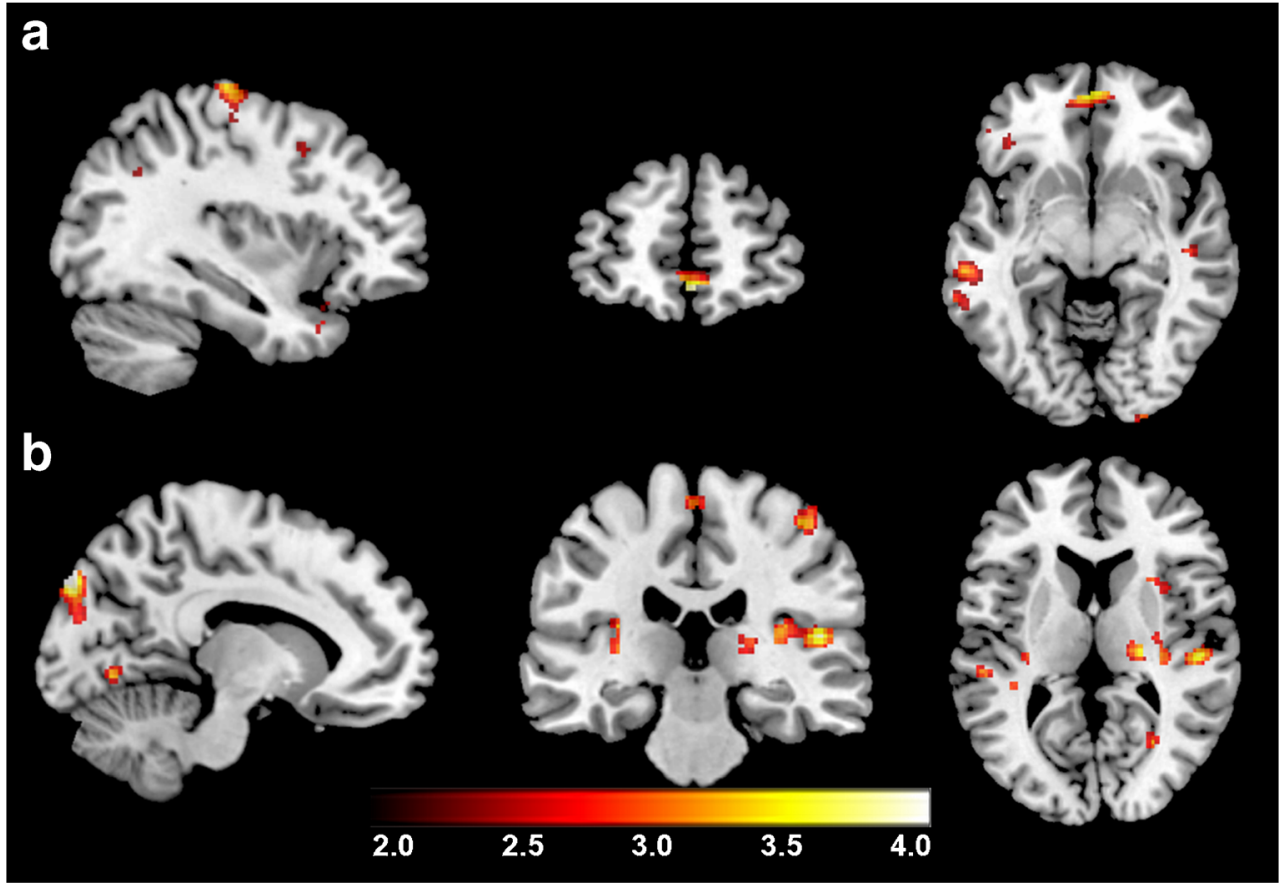

(mainly hyper activation) of frontal, parietal and temporal cortices, basal ganglia, amygdala, cingulate cortex, and cerebellum.

The complex network of brain responses to the activation of the defensive system in OCD may be associated with impairments with the inhibitory response. Contemporary models of OCD pathogenesis acknowledge that two corticalsubcortical pathways may be involved in the failure of the inhibitory systems: (a) the frontostriatal loop (dorsolateralcaudate-striatum-thalamus) responsible for failures in behavioral inhibition and; (b) the orbitofrontal loop (orbitofrontal, medial prefrontal and cingulate) responsible for failures in cognitive inhibitory processes (Aouizerate et al. 2004; Friedlander and Desrocher 2006). Additionally, recent reviews have suggested abnormalities in the ventral orbitostriatal and dorso-fronto-striatal connections in OCD, which may mediate the emotional (i.e., ventral) and the cognitive (i.e. dorsal) symptoms (c.f. Kwon et al. 2009). In fact, in the present study, both of these pathways were found to be hyperactive in patients with OCD for the defensive condition (e.g., medial orbitofrontal frontal; putamen).

Our findings demonstrate that triggering the defensive systems in OCD significantly increased the activation of the temporal regions (e.g., superior and middle), which is consistent with previous research showing that exposure to stimuli provocative of OCD symptoms induces activation of the anterior, lateral, and medial regions of the temporal cortex (Adler et al. 2000). Indeed, van den Heuvel et al. (2009) found that morphological abnormalities of the temporal cortex were particularly evident in patients with "harm-checking" symptoms. These authors speculated that repeated checking might be associated with compensatory activation of the temporal regions due to evidence of non-verbal memory deficits among checkers. Patients with temporal lobe disorders (i.e., frontotemporal dementia and temporal epilepsy) often exhibit such compulsive behaviors. For example, Rosso et al. (2001) found significant incidence of compulsion in patients with frontotemporal and temporal atrophy.

Contrary to our initial hypothesis on the existence of dissociation between anterior and posterior brain regions in the activation of the defensive system, we did not find activations in the visual areas. Emotional activation has been found to be associated with the enhancement of visual perception (Phelps et al. 2006), particularly in the ventral visual stream (Wright et al. 2004), with evidence of an increased amygdala - visual cortex (extra striate and inferotemporal) functional connectivity for both pleasant and unpleasant stimuli (Sabatinelli et al. 2005; Wendt et al. 2011). In particular, several studies have revealed the existence of visual processing impairments in OCD, such as visual memory/visuomotor speed set shifting (Rampacher et al. 2010), body posture discrimination (Shin et al. 2013), and perception of biological movement (Kim et al. 2008). In the present study, we observed that the activation of the defensive system was not associated with activity of the visual system, suggesting that patients with OCD are more sensible to threatening stimuli, rather to its physical characteristics. Consistent with our data, Shapira et al. (2003) did not find any differences in brain activation between patients with OCD and controls for threatening stimuli.

Regarding triggering the appetitive system by the presentation of pleasant (i.e., erotic) IAPS pictures, patients with OCD manifested decreased brain activity in a major posterior 
visual processing region (i.e., superior occipital gyrus). When we selected the comparisons between the high arousal appetitive stimuli and the neutral stimuli, a decreased activation in patients with OCD was witnessed in the frontal cortex (e.g., right pre central gyrus and left para central lobule), and temporo-occipital areas (e.g., left superior occipital gyrus, left fusiform gyrus).

Overall, these results suggest decreased activation mainly in the posterior visual regions for the appetitive condition in OCD, but also in several frontal areas. Results for the overall appetitive condition showed that this deactivation was associated with the superior occipital gyrus. The existence of a potential impairment of the visual system while processing triggers of the appetitive system was already suggested in a study by Olatunji et al. (2011) in which patients with OCD revealed difficulties in disengaging from erotic images during an emotional attentional blink paradigm. Interestingly, Yamanishi et al. (2009) reported that increased regional cerebral blood flow in visual regions (e.g., fusiform gyrus; angular gyrus) was associated with good therapeutic response in OCD patients.

However, other studies have found a deactivation of visual regions for different types of emotional stimuli. For example, Shapira et al. (2003), found decreased activity in the visual cortex for disgust inducing stimuli in OCD. Mataix-Cols et al. (2004) reported decreased lingual activity in response to washing and checking provocation stimuli, which was associated with OCD checking symptoms. This data suggests that the effects of deactivation may not be specific to appetitive survival stimuli but, possibly, be part of a more general response pattern when patients with OCD are confronted with stimuli that activate self-related emotions (e.g. contamination disgust, checking - doubt; erotic - guilt).

fMRI studies of brain activation during the presentation of erotic stimuli with healthy subjects have shown significant activations of the same brain regions that were found to be deactivated in our OCD patients. For example, Kim et al. (2006) presented 10 heterosexual healthy males with film clips of relaxing scenes, sports, and sexually arousing erotic scenes. The authors found that the sexual arousal stimuli were associated with an activation of the occipitotemporal area, anterior cingulate gyrus, insula, orbitofrontal cortex, and caudate nucleus.

In summary, the present study indicates the possibility of dissociation in the activity of the defensive and appetitive survival systems in OCD. Patients with OCD seem to react to basic threat with a strong activation of the defensive system mobilizing many of the brain network systems. In an opposite way, activation of the appetitive system is blocked when facing positive emotional triggers (i.e. erotic images) from the initial stages of visual processing.

Despite the promising nature of these results, generalizations of the current findings need to be approached carefully given several methodological limitations. First of all, the small sample size may have interfered with statistical power, forcing us to less restrictive in terms of statistical criteria.

Secondly, the present study used standardized emotional stimuli (e.g., IAPS) without a cross validation for self-report and autonomic measures. However, the existence of abnormalities in OCD patients' processing of standardized stimuli (valence, arousal, and dominance) was recently shown in a study by Casado et al. (2011), in which patients with OCD were asked to assess, using Lang's dimensional model of emotions, the valence, arousal, and dominance levels of 60 IAPS pictures (pleasant, neutral, and unpleasant) and 100 emotional pictures associated with obsessive-compulsive symptoms. Patients with OCD were found to rate the pleasant IAPS images as less pleasant when compared with controls. As expected, the pictures associated with OCD symptoms were rated as more unpleasant by the OCD group. Additionally, the emotions associated with pleasant and neutral stimuli along with OCD images, were perceived as triggering a lower sense of control (i.e., less dominance).

Additionally, distinct OCD endophenotypes (e.g., washers, hoarders, checkers) as well as different types of risk (genetic risk versus environmental) were reported to be associated with specific brain activation patterns (Mataix-Cols et al. 2004; den Braber et al. 2010). By not differentiating the response in terms of OCD symptomatic profile and genetic risk, the present results were averaged across what may have been a compound of distinct responses. Finally, the use of a $20 \mathrm{~s}$ epoch for stimuli presentation was probably too long and susceptible to processes of emotional habituation and/or desensitization. Future studies, need to take these limitations into consideration and try to replicate the current finding with larger samples, differentiating OCD subtypes, and testing the time course of the emotional responses to these and, eventually, more ecologically valid stimuli (e.g., film clips).

\section{References}

Adler, C. M., McDonough-Ryan, P., Sax, K. W., Holland, S. K., Arndt, S., \& Strakowski, S. M. (2000). fMRI of neuronal activation with symptom provocation in unmedicated patients with obsessive compulsive disorder. Journal of Psychiatric Research, 34(4-5), 317324.

Amaral, D. G., Behniea, H., \& Kelly, J. L. (2003). Topographic organization of projections from the amygdala to the visual cortex in the macaque monkey. Neuroscience, 118(4), 1099-1120.

Aouizerate, B., Guehl, D., Cuny, E., Rougier, A., Bioulac, B., Tignol, J., \& Burbaud, P. (2004). Pathophysiology of obsessive-compulsive disorder: a necessary link between phenomenology, neuropsychology, imagery and physiology. Progress in Neurobiology, 72(3), 195-221. doi:10.1016/j.pneurobio.2004.02.004. 
Beck, A. T., Steer, R. A., \& Garbin, M. G. (1988). Psychometric properties of the beck depression inventory. Clinical Psychology Review, $8(1), 77-100$.

Bloch, M. H., Landeros-Weisenberger, A., Rosario, M. C., Pittenger, C., \& Leckman, J. F. (2008). Meta-analysis of the symptom structure of obsessive-compulsive disorder. The American Journal of Psychiatry, 165(12), 1532-1542. doi:10.1176/appi.ajp.2008. 08020320.

Bradley, M. M., Codispoti, M., Cuthbert, B. N., \& Lang, P. J. (2001). Emotion and motivation I: defensive and appetitive reactions in picture processing. Emotion, 1(3), 276-298.

Bradley, M. M., Codispoti, M., \& Lang, P. J. (2006). A multi-process account of startle modulation during affective perception. Psychophysiology, 43(5), 486-497. doi:10.1111/j.1469-8986.2006.00412.x.

Casado, Y., Cobos, P., Godoy, A., Machado-Pinheiro, W., \& Vila, J. (2011). Emotional processing in obsessive-compulsive disorder. Journal of Anxiety Disorders, 25(8), 1068-1071. doi:10.1016/j. janxdis.2011.07.003.

Del Casale, A., Kotzalidis, G. D., Rapinesi, C., Serata, D., Ambrosi, E., Simonetti, A., Pompili, M., Ferracuti, S., Tatarelli, R., \& Girardi, P. (2011). Functional neuroimaging in obsessive-compulsive disorder. Neuropsychobiology, 64(2), 61-85. doi:10.1159/000325223.

den Braber, A., van't Ent, D., Cath, D. C., Wagner, J., Boomsma, D. I., \& de Geus, E. J. (2010). Brain activation during cognitive planning in twins discordant or concordant for obsessive-compulsive symptoms. Brain : a journal of neurology, 133(10), 3123-3140. doi:10. 1093/brain/awq229.

First, M. B., Spitzer, R. L., Gibbon, M., \& Wiliams, J. B. W. (1996). Structured clinical interview for DSM-IV axis I disorders, clinician version ( $S C I D-C V$ ). Washington: American Psychiatric Press.

Friedlander, L., \& Desrocher, M. (2006). Neuroimaging studies of obsessive-compulsive disorder in adults and children. Clinical Psychology Review, 26(1), 32-49. doi:10.1016/j.cpr.2005.06.010.

Goodman, W. K., Price, L. H., Rasmussen, S. A., Mazure, C., Fleischmann, R. L., Hill, C. L., Heninger, G. R., \& Charney, D. S. (1989). The Yale-brown obsessive compulsive scale. I. Development, use, and reliability. Archives of General Psychiatry, 46(11), 1006-1011.

Kim, S. W., Sohn, D. W., Cho, Y. H., Yang, W. S., Lee, K. U., Juh, R., Ahn, K. J., Chung, Y. A., Han, S. I., Lee, K. H., Lee, C. U., \& Chae, J. H. (2006). Brain activation by visual erotic stimuli in healthy middle aged males. International Journal of Impotence Research, 18(5), 452-457. doi:10.1038/sj.ijir.3901449.

Kim, J., Blake, R., Park, S., Shin, Y. W., Kang, D. H., \& Kwon, J. S. (2008). Selective impairment in visual perception of biological motion in obsessive-compulsive disorder. Depression and Anxiety, 25(7), E15-25. doi:10.1002/da.20402.

Kwon, J. S., Jang, J. H., Choi, J. S., \& Kang, D. H. (2009). Neuroimaging in obsessive-compulsive disorder. Expert Review of Neurotherapeutics, 9(2), 255-269. doi:10.1586/14737175.9.2.255.

Lang, P. J., \& Bradley, M. M. (2013). Appetitive and defensive motivation: goal-directed or goal-determined? Emotion review : journal of the International Society for Research on Emotion, 5(3), 230-234. doi:10.1177/1754073913477511.

LeDoux, J. (2012). Rethinking the emotional brain. Neuron, 73(4), 653 676. doi:10.1016/j.neuron.2012.02.004.

Mataix-Cols, D., Wooderson, S., Lawrence, N., Brammer, M. J., Speckens, A., \& Phillips, M. L. (2004). Distinct neural correlates of washing, checking, and hoarding symptom dimensions in obsessive-compulsive disorder. Archives of General Psychiatry, 61(6), 564-576. doi:10.1001/archpsyc.61.6.564.

Olatunji, B. O., Ciesielski, B. G., \& Zald, D. H. (2011). A selective impairment in attentional disengagement from erotica in obsessivecompulsive disorder. Progress in Neuro-Psychopharmacology \& Biological Psychiatry, 35(8), 1977-1982. doi:10.1016/j.pnpbp. 2011.07.005
Oldfield, R. C. (1971). The assessment and analysis of handedness: the Edinburgh inventory. Neuropsychologia, 9(1), 97-113.

Phelps, E. A., Ling, S., \& Carrasco, M. (2006). Emotion facilitates perception and potentiates the perceptual benefits of attention. Psychological Science, 17(4), 292-299. doi:10.1111/j.1467-9280. 2006.01701.x.

Radomsky, A. S., \& Alcolado, G. M. (2010). Don't even think about checking: mental checking causes memory distrust. Journal of Behavior Therapy and Experimental Psychiatry, 41, 345-351.

Rampacher, F., Lennertz, L., Vogeley, A., Schulze-Rauschenbach, S., Kathmann, N., Falkai, P., \& Wagner, M. (2010). Evidence for specific cognitive deficits in visual information processing in patients with OCD compared to patients with unipolar depression. Progress in Neuro-Psychopharmacology \& Biological Psychiatry, 34(6), 984-991. doi:10.1016/j.pnpbp.2010.05.008.

Rosso, S. M., Roks, G., Stevens, M., de Koning, I., Tanghe, H. L. J., Kamphorst, W., Ravid, R., Niermeijer, M. F., \& van Swieten, J. C. (2001). Complex compulsive behaviour in the temporal variant of frontotemporal dementia. Journal of Neurology, 248(11), 965-970.

Rotge, J. Y., Guehl, D., Dilharreguy, B., Cuny, E., Tignol, J., Bioulac, B., Allard, M., Burbaud, P., \& Aouizerate, B. (2008). Provocation of obsessive-compulsive symptoms: a quantitative voxel-based metaanalysis of functional neuroimaging studies. Journal of psychiatry \& neuroscience : $J P N, 33(5), 405-412$.

Rotge, J. Y., Langbour, N., Guehl, D., Bioulac, B., Jaafari, N., Allard, M., Aouizerate, B., \& Burbaud, P. (2010). Gray matter alterations in obsessive-compulsive disorder: an anatomic likelihood estimation meta-analysis. Neuropsychopharmacology : official publication of the American College of Neuropsychopharmacology, 35(3), 686691. doi:10.1038/npp.2009.175.

Sabatinelli, D., Bradley, M. M., Fitzsimmons, J. R., \& Lang, P. J. (2005). Parallel amygdala and inferotemporal activation reflect emotional intensity and fear relevance. NeuroImage, 24(4), 1265-1270. doi: 10.1016/j. neuroimage.2004.12.015.

Sabatinelli, D., Lang, P. J., Bradley, M. M., Costa, V. D., \& Keil, A. (2009). The timing of emotional discrimination in human amygdala and ventral visual cortex. The Journal of neuroscience : the official journal of the Society for Neuroscience, 29(47), 14864-14868. doi: 10.1523/JNEUROSCI.3278-09.2009.

Sabatinelli, D., Fortune, E. E., Li, Q., Siddiqui, A., Krafft, C., Oliver, W. T., Beck, S., \& Jeffries, J. (2011). Emotional perception: metaanalyses of face and natural scene processing. NeuroImage, 54(3), 2524-2533. doi:10.1016/j.neuroimage.2010.10.011.

Shapira, N. A., Liu, Y., He, A. G., Bradley, M. M., Lessig, M. C., James, G. A., Stein, D. J., Lang, P. J., \& Goodman, W. K. (2003). Brain activation by disgust-inducing pictures in obsessive-compulsive disorder. Biological Psychiatry, 54(7), 751-756.

Shin, N. Y., Jang, J. H., Kim, H. S., Shim, G., Hwang, J. Y., Kim, S. N., \& Kwon, J. S. (2013). Impaired body but not face perception in patients with obsessive-compulsive disorder. Journal of Neuropsychology, 7, 58-71.

Tzourio-Mazoyer, N., Landeau, B., Papathanassiou, D., Crivello, F., Etard, O., Delcroix, N., Mazoyer, B., \& Joliot, M. (2002). Automated anatomical labeling of activations in SPM using a macroscopic anatomical parcellation of the MNI MRI single-subject brain. NeuroImage, 15(1), 273-289. doi:10.1006/nimg.2001.0978.

van den Heuvel, O. A., Remijnse, P. L., Mataix-Cols, D., Vrenken, H., Groenewegen, H. J., Uylings, H. B., van Balkom, A. J., \& Veltman, D. J. (2009). The major symptom dimensions of obsessivecompulsive disorder are mediated by partially distinct neural systems. Brain : a journal of neurology, 132(Pt 4), 853-868. doi:10. 1093/brain/awn267.

Wendt, J., Weike, A. I., Lotze, M., \& Hamm, A. O. (2011). The functional connectivity between amygdala and extra striate visual cortex activity during emotional picture processing depends on stimulus novelty. Biological Psychology, 86, 203-209. 
Williams, L. M., Das, P., Liddell, B. J., Kemp, A. H., Rennie, C. J., \& Gordon, E. (2006). Mode of functional connectivity in amygdala pathways dissociates level of awareness for signals of fear. The Journal of neuroscience : the official journal of the Society for Neuroscience, 26(36), 9264-9271. doi:10.1523/JNEUROSCI. 1016-06.2006.

Wright, P., He, G., Shapira, N. A., Goodman, W. K., \& Liu, Y. (2004). Disgust and the insula: fMRI responses to pictures of mutilation and contamination. Neuroreport, 15(15), 23472351

Yamanishi, T., Nakaaki, S., Omori, I. M., Hashimoto, N., Shinagawa, Y., Hongo, J., Horikoshi, M., Tohyama, J., Akechi, T., Soma, T., Iidaka, T., \& Furukawa, T. A. (2009). Changes after behavior therapy among responsive and nonresponsive patients with obsessivecompulsive disorder. Psychiatry Research, 172(3), 242-250. doi: 10.1016/j.pscychresns.2008.07.004. 